\title{
Exploring latent states of problem-solving competence using hidden Markov model on process data
}

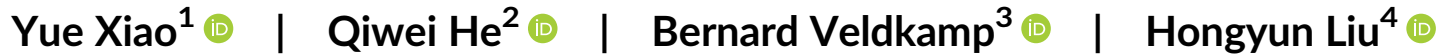

${ }^{1}$ Faculty of Psychology, Beijing Normal University, Beijing, China

${ }^{2}$ Educational Testing Service, Princeton, New Jersey, USA

${ }^{3}$ Department of Research Methodology, Measurement and Data Analysis, University of Twente, Enschede, The Netherlands

${ }^{4}$ Beijing Key Laboratory of Applied Experimental Psychology, Faculty of Psychology, Beijing Normal University, Beijing, China

\section{Correspondence}

Hongyun Liu, Beijing Key Laboratory of Applied Experimental Psychology, Faculty of Psychology, Beijing Normal University, Beijing, 100875, China.

Email: hyliu@bnu.edu.cn

Funding information

National Natural Science Foundation of China; National Science Foundation

\begin{abstract}
The response process of problem-solving items contains rich information about respondents' behaviours and cognitive process in the digital tasks, while the information extraction is a big challenge. The aim of the study is to use a data-driven approach to explore the latent states and state transitions underlying problemsolving process to reflect test-takers' behavioural patterns, and to investigate how these states and state transitions could be associated with test-takers' performance. We employed the Hidden Markov Modelling approach to identify test takers' hidden states during the problem-solving process and compared the frequency of states and/or state transitions between different performance groups. We conducted comparable studies in two problem-solving items with a focus on the US sample that was collected in PIAAC 2012, and examined the correlation between those frequencies from two items. Latent states and transitions between them underlying the problemsolving process were identified and found significantly different by performance groups. The groups with correct responses in both items were found more engaged in tasks and more often to use efficient tools to solve problems, while the group with incorrect responses was found more likely to use shorter action sequences and exhibit hesitative behaviours. Consistent behavioural patterns were identified across items. This study demonstrates the value of data-driven based HMM approach to better understand respondents' behavioural patterns and cognitive transmissions underneath the observable action sequences in complex problem-solving tasks.
\end{abstract}

\section{KEYWORDS}

behavioural pattern, hidden Markov model, PIAAC, problem-solving skills, process data

\section{1 | INTRODUCTION}

Growing interests in assessing problem-solving skills and knowledge in technology-related environments have been facilitated by the move toward delivering assessments via computer and the internet (Bennett et al., 2007, 2010). The use of computers as the delivery platform enables the development of new and innovative item types

Yue Xiao, Faculty of Psychology, Beijing Normal University; Qiwei He, Educational Testing Service (ETS); Bernard Veldkamp, Department of Research Methodology, Measurement and Data Analysis, University of Twente; Hongyun Liu, Beijing Key Laboratory of Applied Experimental Psychology, Faculty of Psychology, Beijing Normal University. (i.e., interactive scenario-based items) and the collection of a broader range of records in log files. The new data source provides information not just on whether test takers are able to solve tasks but how they approach the solution and how much time their efforts take (He \& von Davier, 2016).

As a test-taker attempts an interactive item, his/her actions are recorded, in the form of an ordered sequence of multi-type, timestamped events. This sort of data contained in log files, referred to as process data in this paper, provides information beyond response data that typically show correctness or incorrectness only. This additional information holds promise to help us understand the strategies that 
underlie proficient performance and identify key actions that lead to success or failure (e.g., Arieli-Attali et al., 2019; Han et al., 2019; He \& von Davier, 2015, 2016; Liao et al., 2019).

The information extracted from process data is particularly valuable when examining interactive problem-solving tasks (He et al., 2019, 2021). The analysis of process data has recently gained strong interests, with a wide range of model- and data- driven methods proposed to understand the types of strategies that contribute to successful and unsuccessful problem-solving, identify the behavioural differences between observed and latent subgroups and assess the proficiency on the trait of interests (e.g., Goldhammer et al., 2013; He et al., 2019, 2021; He \& von Davier, 2016; LaMar, 2018; Tang et al., 2020).

However, to extract underlying information from process data, one has to confront a couple of challenges: First, problem-solving strategies are usually not well defined. Even though item developers and content experts could pre-define optimal strategies that are the most efficient ways to solve the tasks, the strategies may not be exclusively recorded in advance (He et al., 2019). Also, there are always unpredictable behaviour patterns in the incorrect response group (Ulitzsch et al., 2021; Ulitzsch, He, \& Pohl, 2021). Second, process data are generally noisy, highly variable, and hard to perceive (Tang et al., 2020). The loose structure of process data may bring issues for model fit and interpretations. Third, to accomplish a problem-solving task usually requires several steps or stages and identifying these stages from each sequence is helpful for understanding test-takers' strategies (Kroehne \& Goldhammer, 2018). However, the segmentation of the observed process usually requires human judgement, which is very time-consuming and laborious.

In this study, we implemented an application from the latent sequence analysis to divide the complex problem-solving process into several task states via a data-driven approach. The aim of this paper is two-fold: first, to explore the latent states and state transitions underlying problem-solving process to reflect test-takers' behavioural patterns, and second, to investigate how these states and state transitions could be associated with test-takers' performance. Specifically, we applied the hidden Markov model (HMM) on action sequences from two items in the Problem-Solving Technology-Rich Environment (PSTRE) domain in the Programme for the International Assessment of Adult Competencies (PIAAC) and compared the results between correct and incorrect groups to characterize the problemsolving processes of different performance groups. A correlation study was performed to investigate the consistency of behavioural patterns across items.

\subsection{PIAAC and PSTRE}

As the largest and most innovative international assessment of adult skills ever conducted, the PIAAC, organized by the Organization for Economic Co-operation and Development (OECD), has been administered in over 40 countries targeting adult populations, ranging from age 16 to 65 (Schleicher, 2008). There are three core assessment domains in PIAAC-literacy, numeracy, and PSTRE. A comprehensive background survey was also included to assist in understanding the interest, attitude, and ability of individuals to appropriately use sociocultural tools-including digital technology and communication toolsto access, manage, integrate, and evaluate information, construct knowledge, and communicate with others (OECD, 2011).

Of the three core assessment domains, the PSTRE domain involves the most interactive item types and is available only on computer-based assessment (CBA). Respondents were routed to the CBA only if they self-reported having some prior computer experience and passed the ICT core test (including the core ICT screening and CBA core test which consisted of 3 literacy and 3 numeracy items) (OECD, 2012).

Participants in the PSTRE domain fall within our research interests. In PIAAC, the PSTRE items were used to assess the ability to "use digital technology, communication tools, and networks to acquire and evaluate information, communicate with others and perform practical tasks" (OECD, 2009). The items assessed adults' competencies in setting appropriate goals and plans, as well as accessing and making use of information through computers and networks (OECD, 2009). The assessment of PSTRE required individuals to engage with complex tasks: for instance, they were required to click buttons or links, select from dropdown menus, drag and drop, copy and paste, and so on. These multitudes of different actions (i.e., all interactions between the respondent and the testing platform) were recorded in log files.

Figure 1 exhibits an example PSTRE item in PIAAC. The layout of this item is simple and straightforward. To solve this problem, test takers are required to fill in the box by performing calculations based on the table provided. Respondents must monitor progress as they select files, checking against the provided criteria to know when they have satisfied the constraints presented in the problem. Sorting the songs by size would greatly facilitate the finding of the solution, but respondents could also solve the items using other procedures. The sorting function can be used either through the dropdown menu or clicking on the shortcut button on the smart portal.

\subsection{Leveraging process data to understand problem-solving skills}

Compared with traditional tests, in which a univariate response is observed for each item, detailed response process records from computer-based tests contain much richer information concerning the respondents. Dynamic records of actions generated during the itemresponse process form a distinct sequence derived from test-taker input. Appropriate analysis of these process/sequence responses promotes the understanding of how people plan, evaluate, and select operations to achieve certain problem-solving goals. This advance in technology offers a great opportunity to enhance educational assessment and presents great challenges, both conceptually and technically, to education researchers (Tang et al., 2020).

The existing methods that engage in extracting meaningful information from complex process data in educational assessments could be 


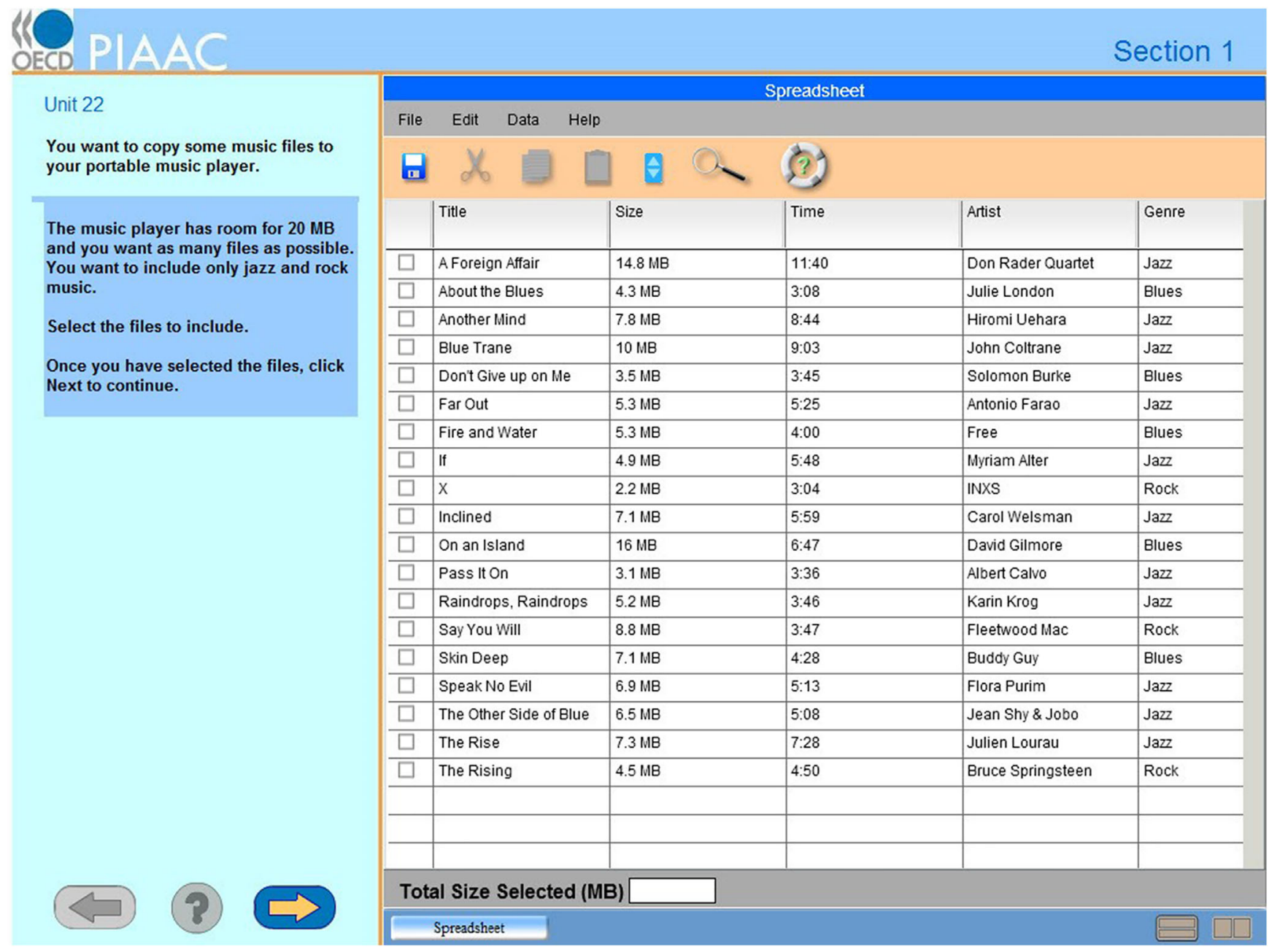

FIG URE 1 An example item with a single environment used in the PIAAC PSTRE test (OECD, 2012) [Colour figure can be viewed at wileyonlinelibrary.com]

roughly classified into three categories: feature-based approach, grambased approach, and sequence-based approach. The feature-based approach often starts with generating features or deriving variables from certain log events from process data (Goldhammer et al., 2017), such as the number of tool usage on one page, or time spent on one webpage. A typical example of a generated feature is the "vary one thing at a time" strategy (VOTAT) generated in problem solving items in Programme for International Student Assessment (PISA) 2012 (Greiff et al., 2015). In this study, a behavioural indicator-whether the respondent followed a VOTAT strategy or not-was found to be highly correlated with correctness in solving the task.

The gram-based approach on process data mainly refers to disassembling a long action sequence into mini-sequences with $n$-grams (i.e., unigrams, bigrams, trigrams, and even higher-order) in each unit. The grams are generally assumed to be independent of each other while the elements within the gram (e.g., bigram) may show the dependence when taking the action co-occurrence and order into account. This approach is sourced from the $n$-gram linguistic feature representative method (Damashek, 1995) in natural language processing. He and von Davier $(2015,2016)$ first introduced the n-gram model to extract sequence patterns from process data in one PSTRE item and determined the most robust grams using the chi-square feature selection method (Oakes et al., 2001) to differentiate behaviours of successful and unsuccessful test-takers during the problem-solving process. Given the straightforwardness in deriving features from observed sequences, $\mathrm{n}$-gram based approaches have been often employed in recent studies related to process data (e.g., Han et al., 2019; Liao et al., 2019; Stadler et al., 2019). These studies demonstrate the effectiveness of the $n$ gram method in analyzing process data and comparing behavioural patterns across groups. However, this method unavoidably divides the action sequence into independent pieces. While the order of observed actions is partly retained within $n$-gram based mini-sequences, the entire behavioural process is often broken down and information may not be completely preserved (He et al., 2019).

The sequence-based approach features in regarding the entire sequence as an input with considerations on local dependencies between actions, which can further be roughly divided into two categories. In the first category of sequence-based analysis, distances between two or multiple sequences are often computed. For instance, He et al. $(2019,2021)$ applied the longest common subsequence 
method (Hirschberg, 1975, 1977; Sukkarieh et al., 2012) to identify how different the action sequence taken by a test taker to solve an item is from the action sequence that subject matter experts consider optimal to solve the problem. The longer the distance from the predefined action sequences, the less likelihood of a correct response. The second category of sequence-based approach is to model the relationship between every two actions according to the input sequences of all respondents, like social network analysis (SNA; Zhu et al., 2016), Bayesian Networks (BNs; Levy, 2014, 2019), and hidden Markov model (HMM; Rabiner, 1989; see also Boyer et al., 2011; Jeong et al., 2008). Biswas et al. (2010) used HMM as a direct probabilistic representation of the internal states and interpret these states in terms of theoretical learning strategies, which facilitated identification, interpretation, and comparison of student learning behaviours at an aggregate level. Like students' mental processes, the states of an HMM are hidden, meaning they cannot be directly observed but produce observable output (e.g., actions in a learning environment).

Among those, Markov and hidden Markov models are increasingly popular in educational measurement (e.g., LaMar, 2018; Li et al., 2016; Wang et al., 2018) and in analyses of process data with serial dependence (e.g., Bergner et al., 2017; Dutilh et al., 2010; Shu et al., 2017). The assumption of the Markov property in the model can describe the dependence between actions. Compared with Markov models, HMMs that were also used in this paper can further capture an underlying layer of unobservable stochastic structures that is thought to influence observations. These structures are referred to task states or behaviour states, and can be related to and interpreted as cognitive states or problemsolving strategies (e.g., Biswas et al., 2010; Boyer et al., 2011).

In educational research, HMM is commonly used in the form of Bayesian Knowledge Tracing (BKT; Corbett \& Anderson, 1994; van de Sande, 2013) to model the student's changing knowledge state during learning process. As a special case of an HMM (Pelánek, 2017), BKT assumes the known or unknown state of each skill as a binary variable. The observations are the outcomes (correct or incorrect) of the student's item responses and each hidden state are the updated skill mastery state of the student at each time node. The state transition probability reveals the probability of learning a skill if the student does not know the skill previously. In the application of this paper, we used only the generic form of HMMs to model respondents' action sequences to one item. The observations in our study are unordered categorical actions and the hidden states may uncover the different stages during the process of solving items, which could indicate respondents' behavioural patterns or characteristics.

\section{3 | The present study}

A problem-solving task usually involves several necessary stages (or subtasks) to achieve the solution. At each stage, test-takers would exhibit different observable behaviours. Finding these stages behind the observed action sequence will help us gain a deeper understanding of test-takers' problem-solving processes. As a specific probabilistic generative model with a "built-in" state transition system,
HMM provides a framework for this. The task states learned in HMM can be treated as different problem-solving stages or subtasks.

Therefore, the present study aims to explore the latent states of test-takers' problem-solving processes underlying the observed action sequences. In particular, we are interested in three research questions: (1) what latent states can be extracted under one PSTRE scenariobased environment and how these latent states are transitioned; (2) whether these latent states can be well reflected by action sequences and validated by predefined problem-solving strategies; and (3) whether the test takers show differences in behaviours and/or engagement by their problem-solving competence in the latent states and whether their behaviour patterns show consistency across items. Given these research objectives, we employed the HMM to identify test takers' hidden states during the problem-solving process and analyzed the behaviour characteristics of different performance groups. We conducted comparable studies in two PSTRE items with a focus on the US sample $(N=1355)$ that was collected in PIAAC 2012.

The remainder of this article is structured as follows. In Section 2, we introduce the instrument, data, theoretical model and application of HMM conducted in this study. In the following section, we present the results from the HMM analysis by showing the latent state plots by each item, comparing the transition paths against predefined problem-solving strategies, and comparing results between performance groups and between items. Finally, we discuss the findings of this study and its generalizability.

\section{2 | METHOD}

\section{1 | Instrument and sample}

A total of 14 items were administrated in the PSTRE assessment by two clusters. Each cluster had seven items, all in a fixed sequence order. Two PSTRE items, U19a and U19b, which located as the first two items in the second PSTRE cluster (PS2) were used in this study. These two items shared a common context (bike club membership) but required respondents to use different strategies to solve the tasks, respectively.

Item U19a involved two environments, a spreadsheet and email. Respondents were asked to find the ID of a particular member from the spreadsheet and then to send it by email to a pre-specified email address. The spreadsheet entailed four columns of information for each club member. The length of the table was sufficient for attracting respondents to use tools (searching or sorting) to facilitate the identification process. This item was dichotomously scored according to whether the correct ID was sent. Item U19b shared the same spreadsheet environment as U19a and involved another word processor environment. The respondents were asked to identify the members who meet multiple conditions in the spreadsheet and mark their records (click checkbox). The information could most efficiently be located within the long spreadsheet by using a sorting function. The information about those conditions was given in the word processor (OECD, 2017). There were four rows satisfying all the conditions, thus recorded as correct answers. To better profile the behavioural 
patterns by respondents with different proficiency levels, we used finer-grained scoring rules by counting the number of correct identification (i.e., 0-no correct row marked, 1-one correct row marked, 2two correct rows marked, 3-three correct rows marked, and 4-total four correct rows marked). ${ }^{1}$ There was no penalty on the incorrect rows marked. It is noted that respondents typically had only one visit to each item. No re-visit allowed when the respondent confirmed to proceed to the next item.

A total of 1355 test-takers of the United States who responded to both items U19a and U19b were focused in this study. Among this PIAAC subsample, $46.5 \%$ of them were male. Approximately half of the respondents attained a level of education higher than a highschool diploma, while the other half, that is, $40.3 \%$ and $9.2 \%$ of the respondents reported the high-school and lower than high school as their highest level of education, respectively.

\subsection{Action sequence}

Action sequences that were collected in U19a and U19b were re-coded in this study, ${ }^{2}$ which resulted in 39 and 47 unique action types for these two items (U19a and U19b), respectively. A list of actions and their corresponding interpretations, as well as their frequencies, are summarized in Appendix A. Based on the recoded actions, the length of observed sequences followed a slightly positively skewed distribution in both items. In U19a, respondents averagely used 8.73 actions (median $=8$ actions), with a minimum of 3 actions and 46 as the maximum. Comparatively, the length of action sequences in U19b followed a sparser distribution with a longer tail in the end of large value. On average, respondents used 19.72 actions (median $=14$ actions) to solve the item, with a minimum 3 actions and 263 as the maximum.

To solve these two PSTRE items, respondents can and are expected to use interactive tools such as searching and sorting engine to speed up identifying key information. A typical example of a testtaker's action sequence in U19a is listed in the box below.

Example: START, SS, SS_TYPE_FN, MC, MC_SEND, NEXT, NEXT_OK.

This action sequence could be interpreted as: the test taker starts an item (START), clicks on the spreadsheet page (SS), and types in the full name of a specified person to execute a search (SS_ TYPE_FN). Then, the test taker switches to the email environment (MC) to fill in the answer and sends the email out (MC_SEND). Finally, the test taker clicks on the forward-arrow button in navigation pane to move on to the next item (NEXT) and confirms the choice in a pop-up dialogue box (NEXT_OK).

\section{3 | Modelling}

HMMs are useful when one can think of underlying events probabilistically generating surface events (Manning \& Schütze, 2002). In an
HMM, each observation in an input sequence is assumed to be generated by a hidden state according to the emission probability distribution of that state. The observations are conditionally independent given the underlying hidden state sequence, and the state sequence is often a first-order Markov chain with a finite number of latent states. Figure 2 shows a time-slice representation of an HMM. Each round node represents a hidden state $S_{t}$ at time point $t$ and each square node denotes an observation $Y_{t}$. The arrows denote the conditional probability that the hidden state evokes the observation or that the previous hidden state transits to the current state. Given the sequence of length $T$, the joint likelihood of observable actions $Y_{1: T}=\left(Y_{1}, Y_{2}, \ldots, Y_{T}\right)$ and latent states $S_{1: T}=\left(S_{1}, S_{2}, \ldots, S_{T}\right)$ can be written as (Ghahramani, 2001):

$$
P\left(Y_{1: T}, S_{1: T}\right)=P\left(S_{1}\right) P\left(Y_{1} \mid S_{1}\right) \prod_{t=2}^{T} P\left(S_{t} \mid S_{t-1}\right) P\left(Y_{t} \mid S_{t}\right)
$$

where $S_{t}$ belongs to a set of $K$ latent states, $P\left(S_{1}\right)$ represents the probability of the initial state, $P\left(Y_{t} \mid S_{t}\right)$ represents the conditional probabilities of the action $Y_{t}$ given the latent state $S_{t}$ (which is called emission probability), and $P\left(S_{t} \mid S_{t-1}\right)$ provides the transition probability between states, independent to the time point $t$. These three types of probability values are the parameters to be estimated by the model given the observed sequences and the number of possible states.

Model estimation can be implemented through the expectationmaximization (EM) algorithm for a given number $K$ of latent states. The HMMs with different number of states can be compared through two model fit indexes, AIC (Akaike information criterion; Akaike, 1973) and BIC (Bayesian information criterion; Konishi et al., 2004). A lower value of AIC or BIC indicates a better model fit, which helps determine the optimal number of states.

Due to the assumption that observable actions are probabilistically emitted by hidden states, given the trained model, the most probable sequence of states underlying an observed action sequence can be found by the Viterbi algorithm (Forney, 1973; Rabiner, 1989; Viterbi, 1967). This algorithm determines the sequence of states which maximizes the likelihood of the joint distribution of the hidden states given the entire observed sequence.

In the current study, the HMM was learned on the action sequences of 1355 respondents in U19a and U19b separately. It was noted that there were 15 missing responses in U19a, thus only 1340 respondents' performance scores were used in analyzing the relationship between the latent states derived from HMM and respondents' problem-solving proficiency in this item. Also, the missing responses were not included in the study to investigate the consistency of behaviours between the two items.

Considering only marginal differences were reported between results with and without sampling weights in previous studies using the same PSTRE item (U19a) in process data analyses (He \& von Davier, 2015, 2016), we did not expect a substantial difference in similar settings in the current study, hence, we conducted the whole set of analyses without sampling weights. 
FIGURE 2 A representation of the HMM. the square nodes denote the observed data sequence while the round nodes represent hidden states

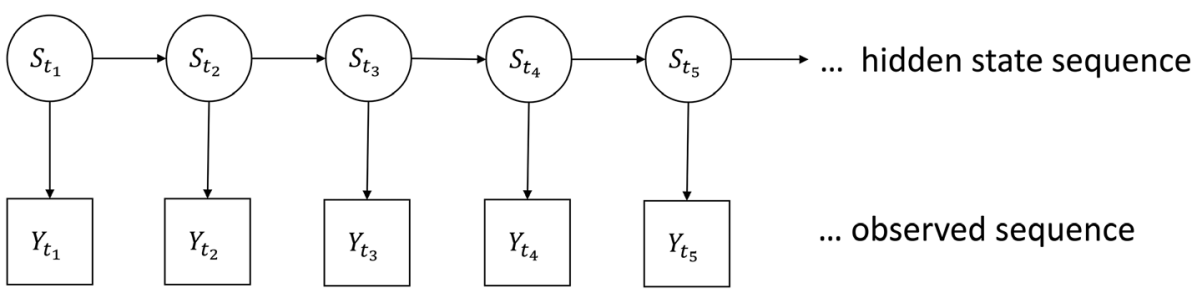

\section{$2.4 \quad$ Analytic procedure}

Given concerns on the different number of unique action types (i.e., 39 and 47 in U19a and U19b respectively) and knowledge on a variety of respondents' underlying strategies learned from the previous studies (He et al., 2019, 2021), we pre-specified the number of states in HMM in a range from 1 to 9 in U19a and a bit broader range from 1 to 12 in U19b because of its higher complexity. The optimal number of hidden states in each item was determined by the meaningful interpretations on the derived model and the common model selection criteria AIC and BIC, where lower values are preferred in both criteria, to assess comparative model fit. All computations of HMM learning on respondents' action sequences were performed using the depmixS4 package (Visser \& Speekenbrink, 2010) in Program R.

The parameter learning task in HMMs is to find, given an output sequence or a set of such sequences, the best set of state transition and emission probabilities. That is, we were interested in the most likely sequence of hidden states that could have produced the output action sequences. This could be evaluated by the joint probability of both the state sequence and the observations for each case. We summarized the frequencies of states and state transitions given each respondent's action sequence per item, and compared the distribution of frequencies of hidden states between different performance groups. Specifically, we used the Wilcoxon rank-sum test (Mann \& Whitney, 1947; Wilcoxon, 1945) to compare the frequency of states and/or state transitions between two groups (correct vs. incorrect) in U19a, and used the Kruskal-Wallis test (Kruskal \& Wallis, 1952), an extension of Wilcoxon rank-sum test among more than two groups, in U19b, where five scoring groups were defined (0-incorrect, 1 to 4 as the number of correct marked entries). The Bonferroni correction for the $p$-value were also performed for comparisons among more than two groups. Because the two PSTRE items U19a and U19b has partially shared similar subtasks, we further computed the Spearman correlation between the state (transition) frequencies to investigate behavioural consistency across items.

\section{3 | RESULTS}

\subsection{Model selection}

We first checked the model fit statistics to ensure the data could be well explained under the HMM with the best optimal number of latent states. The model fit results were summarized in Table 1. Aligned with the principle of model selection of the minimized value from AIC and $\mathrm{BIC}$, a 7-state HMM resulted in the best fit in U19a given its lowest values in the two indexes. More complicated results were found in U19b. Valley points in AIC and BIC were both shown tho statesthe 8-state model and 11-state model. However, the 11-state model was far more challenging in meaningful interpretation than the 8-state model. Therefore, to keep the model simple and interpretable, we selected the 8-state model for U19b, and will only report 8-statemodel results for $\mathrm{U} 19 \mathrm{~b}$ thereafter.

\section{2 | Model interpretation}

Per the first research question, we extracted the hidden states for the two items U19a and U19b by their best fit model (stated above), 7 states and 8 states, respectively. The estimated response and transition model parameters for $\mathrm{U} 19 \mathrm{a}$ and $\mathrm{U} 19 \mathrm{~b}$ are respectively displayed in Appendix B and Appendix $C$ in the form of probabilities. Each hidden state was interpreted based on its emission structure (i.e., response model), as well as the state transition matrix. Following each hidden state, we could get a better understanding on respondents' problem-solving strategies at the group level. To facilitate understanding in a more intuitive way, Figure 3 and Figure 4 show the main results of two learned HMMs in a graphic fashion.

\subsection{1 | Item U19a}

Figure 3 presents the seven hidden states and corresponding actions in item U19a. Each box represents a state (abbreviated as "St" in the figure), and all actions that would be emitted with a probability of greater than 0.1 in that state are listed inside, as well as the corresponding probability values. Arrows indicate transitions between states, and the numbers next to them are the corresponding transition probabilities. For clarity, the transitions with probabilities less than 0.1 are not shown.

When the actions and transitions of the resulting states were inspected from Figure 3 , the following observations could be made for item U19a. The initial state was State 1 with a probability of 1 , and State 1 contained only one action START, so we labelled this state as "Start". State 2 mainly included the correct markup of the required member record (SS_flag_T with the emission probability of 0.571) and the start of searching and sorting engines on spreadsheet page (SS_Se and SS_So with a probability of 0.237 and 0.142 respectively), indicating the "Use of tools". State 3, basically, involved the 


\begin{tabular}{|c|c|c|c|c|c|}
\hline & \multicolumn{2}{|l|}{ U19a } & & \multicolumn{2}{|l|}{ U19b } \\
\hline & AIC & $\mathrm{BIC}$ & & AIC & BIC \\
\hline 1-state model & 59613.32 & 59893.68 & 1-state model & 141341.80 & 141718.70 \\
\hline 2-state model & 51309.88 & 51892.73 & 2-state model & 133351.20 & 134129.60 \\
\hline 3-state model & 45827.79 & 46727.89 & 3-state model & 115480.30 & 116676.50 \\
\hline 4-state model & 42690.44 & 43922.55 & 4-state model & 112235.50 & 113866.00 \\
\hline 5-state model & 39240.01 & 40818.88 & 5-state model & 102098.20 & 104179.30 \\
\hline 6-state model & 39197.58 & 41137.97 & 6-state model & 98452.15 & 101000.30 \\
\hline 7-state model & 34512.75 & 36829.41 & 7-state model & 95427.63 & 98459.20 \\
\hline 8-state model & 35752.81 & 38460.49 & 8-state model & 84521.88 & 88053.25 \\
\hline \multirow[t]{4}{*}{ 9-state model } & 37341.34 & 40454.80 & 9-state model & 87041.94 & 91089.50 \\
\hline & & & 10-state model & 80340.22 & 84920.35 \\
\hline & & & 11-state model & 79598.69 & 84727.77 \\
\hline & & & 12-state model & 80773.64 & 86468.07 \\
\hline
\end{tabular}

TABLE 1 Model fit of HMMs with different numbers of states for U19a and U19b

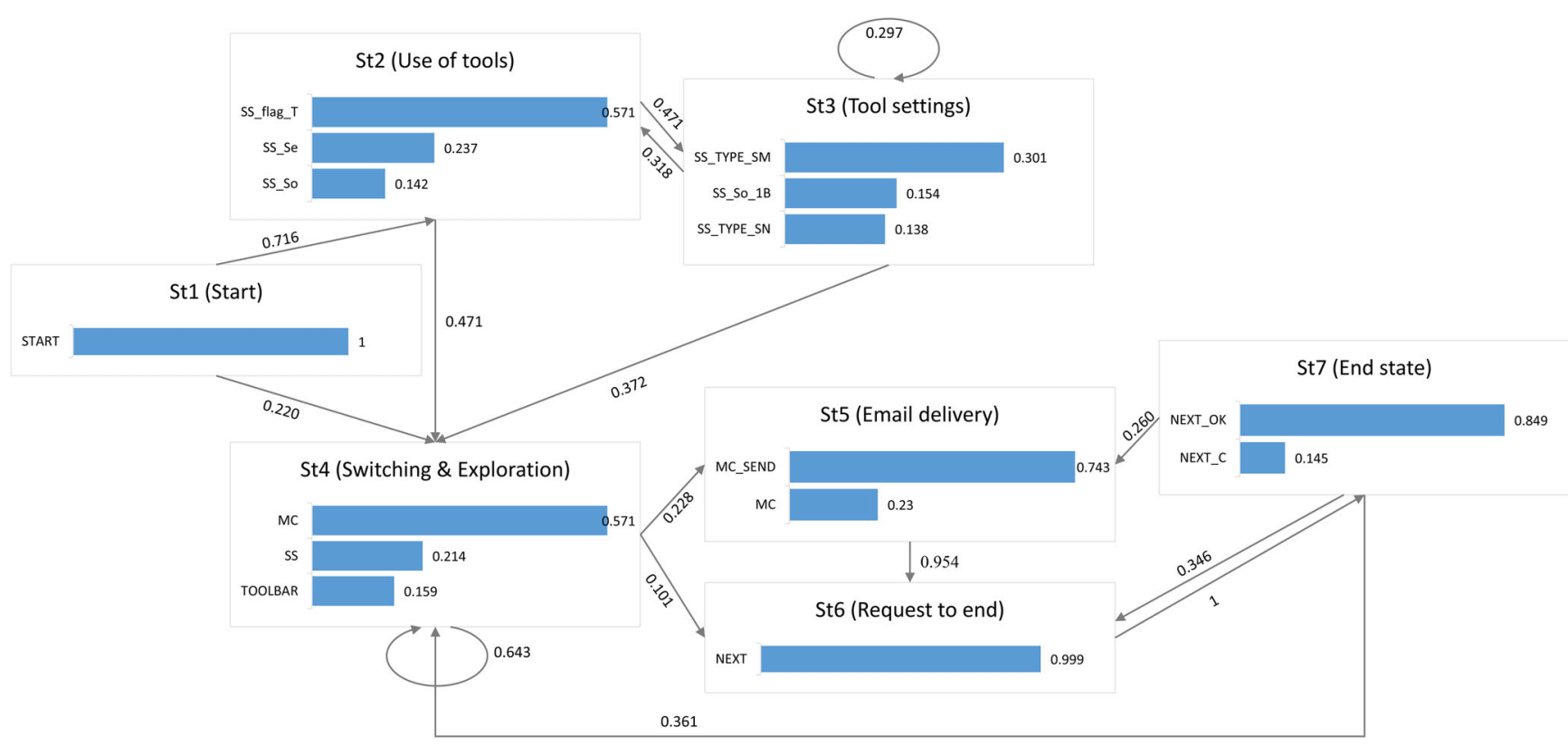

FIGURE 3 A diagram summarizing the main results of the learned HMM for U19a each box represents a state (abbreviated as "St" in the figure, the same below), and all actions that would be emitted with a probability of greater than 0.1 in that state are listed inside, as well as the corresponding probability values. Arrows indicate transitions between states, and the numbers next to them are the corresponding transition probabilities. For clarity, the transitions with probabilities less than 0.1 are not shown [Colour figure can be viewed at wileyonlinelibrary.com]

conditions specified by the respondent for sorting or searching, among which sorting by the second column ("Name") in the first choice (SS_So_1B) and searching with the surname or spelling mistakes (SS_TYPE_SM, SS_TYPE_SN) were more common. We referred to this state as "Tool settings". State 4 indicated environment "Switching and Exploration", as it was dominated by switching to spreadsheet or email pages and clicking on non-useful options in the toolbar. State 5 was dominated by the actions of sending email and switching to the email page, in which the emission probability of the former action was 0.743 , more than three times of the probability of the latter. Therefore, this state could be regarded as a representation of "Email delivery". State 6 emitted NEXT in the probability of 1, representing the "Request to end". State 7 was comprised of the confirmation and cancellation of ending this item, representing a selection state after a respondent clicks on NEXT, so we called it "End state" in this study.

Then we focused on the transition between states with probabilities greater than 0.1 . The transition probability from State 1 to State 2 was 0.716 , over three times of the probability of the transition from State 1 directly to State 4 . This suggested that, after starting the item (State 1), respondents had a high probability of using tools to assist problem solving (State 2), such as flagging the record or starting the 


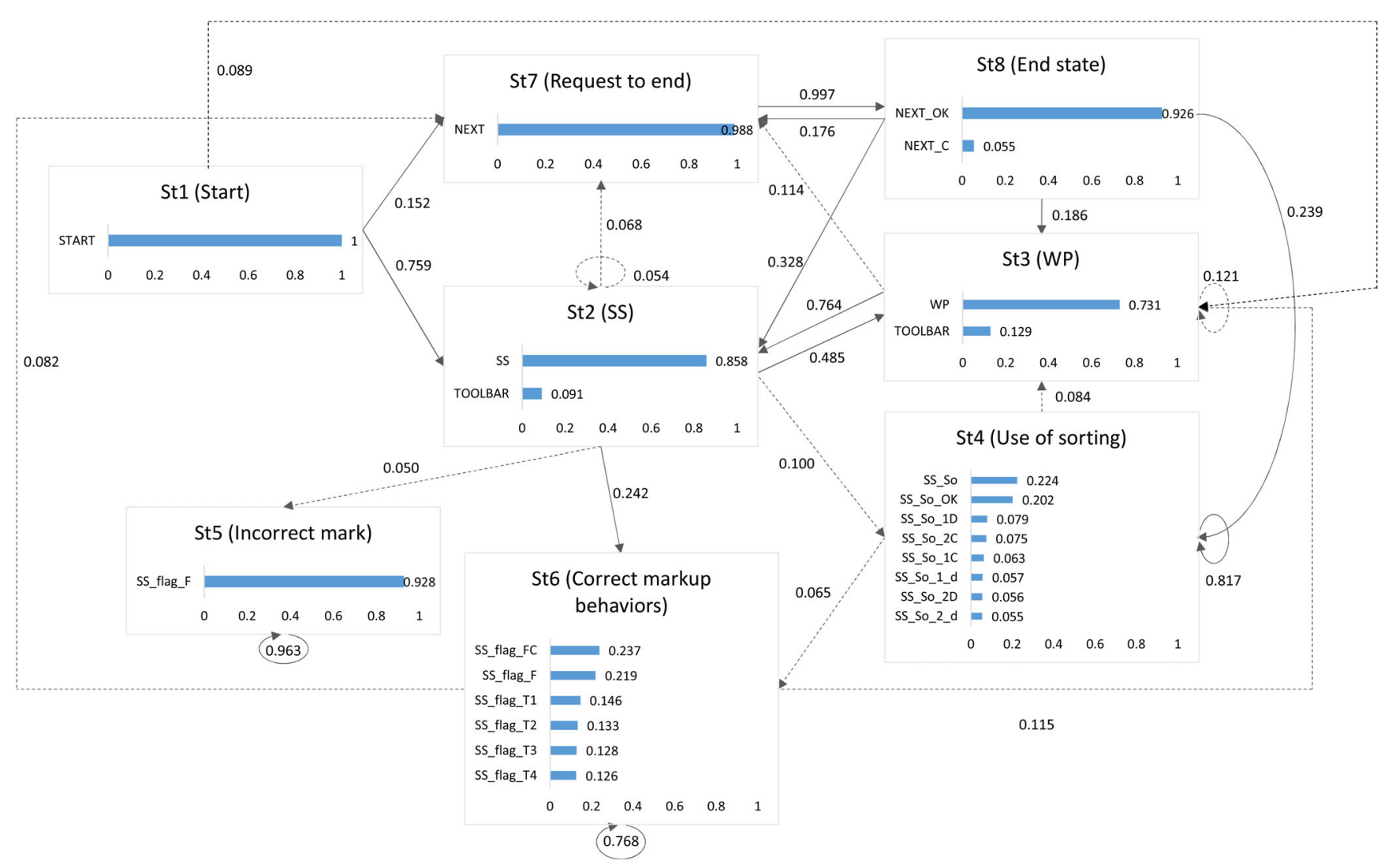

FIGURE 4 A diagram summarizing the main results of the learned HMM for U19b. Only the actions with probability larger than 0.05 are shown in the plot of each state. A solid arrow indicates that the transition probability is greater than 0.15 , a dashed arrow means that the transition probability is between 0.05 and 0.15 , and the transitions with a probability less than 0.05 are not shown [Colour figure can be viewed at wileyonlinelibrary.com]

sorting or searching engine. However, the respondents might also explore different environments before figuring out solutions (State 4). The transitions from State 2 to States 3 and from State 2 to State 4 were both in a moderate probability of 0.471 . The change from State 2 to State 4 mainly indicated that the respondent proceeded to the next step after marking the record. Because State 2 might also be the start of sorting or searching, it was logical that State 2 could be followed by State 3 that represented the settings of sorting or searching. The State 3 could be followed by either State 2 or State 4 or even stay in the same state, and the probabilities of all these transitions were about 0.3 to 0.4 . Staying in State 3 implied that the respondent set more than one sorting condition (since we recoded the three options of sorting as separate actions) or searched more than one time with different keywords. The transition from State 3 to State 2 or State 4 indicated that the respondent moved forward (for example, flagged or switched the environment) after finding an answer; there was also a possibility that the respondent restarted the searching or sorting. As shown in Figure 3, States 1, State 2, and State 3 eventually were converted to State 4 . Among these transitions, the path from State 1 to State 2 (to State 3 and back, if searching or sorting is used) and then to State 4 corresponded to the first half of the correct solution: starting the item, selecting the right member, and switching to the email environment.
As shown in Figure 3, the remaining states following State 4, that is, from State 4 to State 5 , then to State 6, and finally to State 7, corresponded to the second half of the efficient solution: switching to email page, sending the mail, requesting to end the item, and then confirming the end. We examined the original process data and found that respondents usually clicked on MC (switch to email page) or SS (switch to spreadsheet page) several times in a row. This partly explained the high probability $(0.643)$ of State 4 to stay in itself, which was almost three times higher than the probability of transition from State 4 to State 5 . The transition from State 4 to State 5 might also indicate continuous clicks of the MC button as State 5 had a probability of 0.23 to emit MC (switching to email page). These showed the inefficient actions of respondents. The transition from State 4 to State 6 meant that the respondent switched the environment or explored the toolbar and then clicked on NEXT, which had a low probability of 0.101 . This also indicated some inefficient behaviours, or mistakes due to a lack of understanding of the item content or carelessness. After State 6 (Request to end), if the respondent regretted and cancelled in State 7 (End state), it was likely to change to State 4, State 5 , or State 6 in a similar probability around 0.3. The transition from State 7 to State 4 (Switching environment and Exploration) showed that the respondent was unsure about the previous response behaviours and came back to check the information in the spreadsheet. The 
transition from State 7 to State 6 indicated that the respondent requested to end the item again after cancelling the move to the next item. As the probability was 0.346 , it was more likely to represent the hesitation to end the item rather than a remedy for the previous wrong cancellation. However, respondents in this transition did not make new contributions to solve this item. Finally, the transition from State 7 to State 5 possibly suggested the respondent recognized that he/she had not completed a key step (i.e., sending mail).

\subsection{2 | Item U19b}

Item U19b shares similar content with U19a but with a more complex problem-solving procedure to label the key information in request. Analogously to the analysis in item U19a, we present the eight hidden states and corresponding actions in item U19b in Figure 4. Similar as U19a, this item started with an initial state which was labelled as "Start". Both State 2 and State 3 indicated environment switching and exploration because they were dominated by the switch to spreadsheet and word processor pages respectively, with a probability of exceeding 0.7 , and both of them could also emit a non-useful action TOOLBAR with a probability of around 0.1. For convenience, we labelled State 2 and State 3 as "SS" and "WP", respectively. State 4 mainly represented the "Use of sorting", as it involved the start and confirmation of the sorting engine and the specific settings in sorting. Both State 5 and State 6 were related to the mark of records. State 5 specifically reflected the flagging of incorrect records because the emission probability of SS_flag_F exceeded 0.9 , so we labelled it "Incorrect mark". State 6 included incorrect markup (SS_flag_F), correct markup (SS_flag_T1 to SS_flag_T4), and cancellation of incorrect markup (SS_flag_FC). The latter two both belonged to correct markup behaviours, and their emission probabilities added up to more than 0.7. Thus, State 6 could be considered as a representation of "Correct markup behaviors". State 7 could be seen as the "Request to end" since it was dominated by NEXT with the emission probability nearly 1. State 8 was the "End state", which was the same as State 7 in U19a.

According to the task content of U19b, the main part of the optimal sequence could be mapped onto the path from State 1 to State 2 , to State 4 , then to State 6. It corresponded to starting and checking the requirement in the word processor page, switching to spreadsheet page, using sorting to find the correct records, and then marking them. It is noted that the default interface upon starting the item was the word processor page, hence, respondents did not need to deliberately switch environment to word processor, that is, transitioning to State 3 , at the beginning. However, respondents might still click the word processor page immediately after starting the item but with a quite low probability of 0.089 , as shown in Figure 4 . The more likely transitions from the initial state (State 1) were switching to spreadsheet page (State 2 ) with a probability of 0.759 , or directly requiring ending the item (State 7), of which the probability was 0.152. The latter transition indicated an aberrant solving behaviour of a small group of testtakers. The emission probability of transition in both directions between State 2 and State 3 was in a high value, suggesting a common pattern of switching environment to solve this item. Respondents might repeatedly check the requirements on the word processor page when searching for information in the spreadsheet. The sum of the probabilities of transitions from State 2 to State 6 and State 5 (both related to markup behaviours) was about 0.30 , which was higher than the probability of 0.1 from State 2 to State 4 (Use of sorting). This was primarily because respondents might switch back to the spreadsheet multiple times to mark records or change previous marks. Additionally, we noticed that a few respondents did not use sorting function but merely used their eyes to screen for the key information in the long spreadsheet. This also resulted in the transition from State 2 directly to states related to markup behaviours.

The most likely transition from State 4 (Use of sorting) was remaining in itself with a high emission probability of over 0.8 , which implied respondents usually employed the sorting function by multiple times. ${ }^{3}$ The transition from State 4 to State 6 represented an efficient strategy to get the potential correct response, which could be interpreted as the respondents found results in spreadsheet using sorting function, and then correctly marked them. In addition, State 4 and State 6 had relatively low emission probabilities to transit to State 3 , which might show a latent checking behaviour, for instance, a respondent might switch to the word processor to double-check the instruction and the task requirements. Similar as State 4, the most likely transition from both State 5 (incorrect markup) and State 6 (correct markup) also remained in themselves with a high emission probability as 0.963 and 0.768 respectively. It suggested respondents usually adopted multiple trials in flagging correct or incorrect entries.

After the main process of problem-solving, we arrived at the ending states in U19b. The shortest transition sequence from State 6 was to State 7 , finally to State 8 , corresponding to the request to end the item after marking the records, and then a confirmation of the end. However, respondents might also request to end after switching environments, for instance, from States 2 or 3 to State 7 in a quite low probability though with 0.07 and 0.11 respectively), possibly due to the checking or aimless behaviours. The transitions from State 8 (End state) to State 7 (Request to end), or to State 2 (SS) or State 3 (WP) had the similar interpretations as those in results of U19a, revealing hesitative behaviours. However, the transition from State 8 to State 4 might cause an extra attention. After cancelling the request to end the item, the respondent went back to use the sorting function and this transition had a probability of 0.239 . This uncommon path might suggest the possibility of an unintentional click on NEXT.

\section{3 | Comparison of hidden states with expert- predefined sequences}

Per the second research question, we validated the underlying states derived from HMM with the optimal strategies predefined by item designers and experts, which were also regarded as "gold standards" when measuring the similarity between individual problem-solving 
FIGURE 5 Average frequencies of seven states for incorrect and correct groups in U19a. The asterisk indicates that for the state, the frequency difference between the two groups is significant $(p<0.05)$ with the Bonferroni correction under the Wilcoxon test [Colour figure can be viewed at wileyonlinelibrary.com]

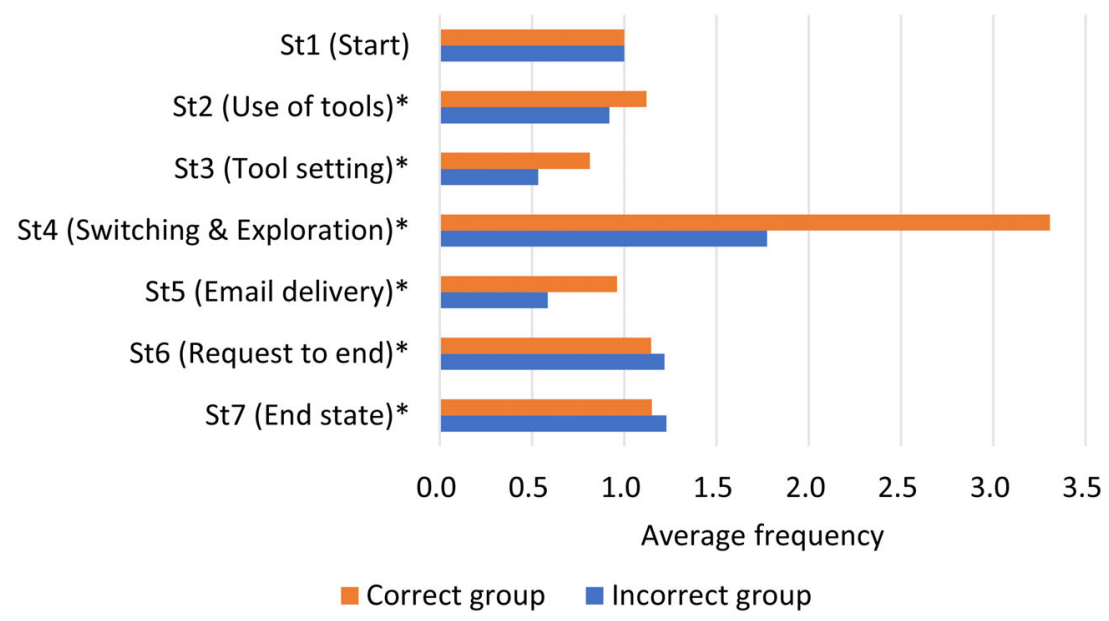

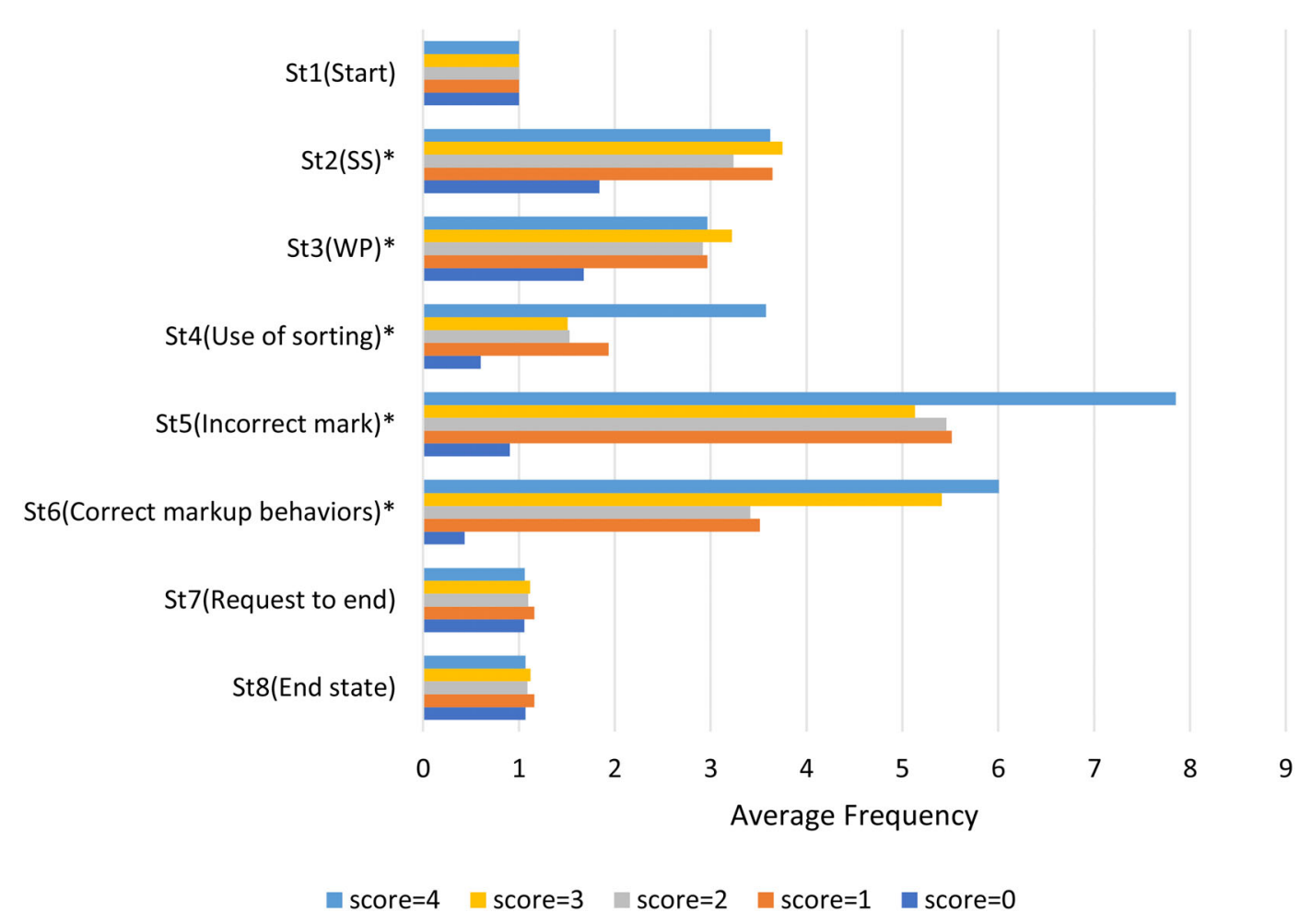

FIGURE 6 Average frequencies of eight states for groups with different scores of U19b. The asterisk indicates that for the state, the frequency difference between the five groups is significant $(p<0.05)$ with the Bonferroni correction under the Kruskal-Wallis test [Colour figure can be viewed at wileyonlinelibrary.com]

sequence and reference sequences (He et al., 2019, 2021). According to the predefined optimal sequences, there were four optimal strategies to solve the item U19a: using searching function through either toolbar or menu and using sorting function through either toolbar or menu. To map onto the latent stages, the core stages to extract key information concentrated on State 2 and State 3 back and forth. The whole predefined optimal strategies were mainly explained by the latent state in sequential order, except that the transition from State 4 was the highest emission probability to itself, implying higher chances for respondents to execute repetitive behaviours in switching and exploration.
The optimal strategy to solve the item U19b could be summarized as: After starting the item, respondents should switch to the spreadsheet page (word processor page is the default opening page), sort the records by two conditions, then mark the correct records, and require and confirm the end of the item. This corresponded to the hidden states with the transition from State 1 to State 8 (i.e., State $1 \rightarrow$ State $2 \rightarrow$ State $4 \rightarrow$ State $6 \rightarrow$ State $7 \rightarrow$ State 8 ). It was noted that State 3 (switch back to word processer environment) and State 5 (incorrect mark) were not compulsory in the predefined strategy, which made a good sense. These two states exhibited the diversity of problemsolving sequences in the sample. 
TAB LE 2 Spearman correlations between state frequencies of two items

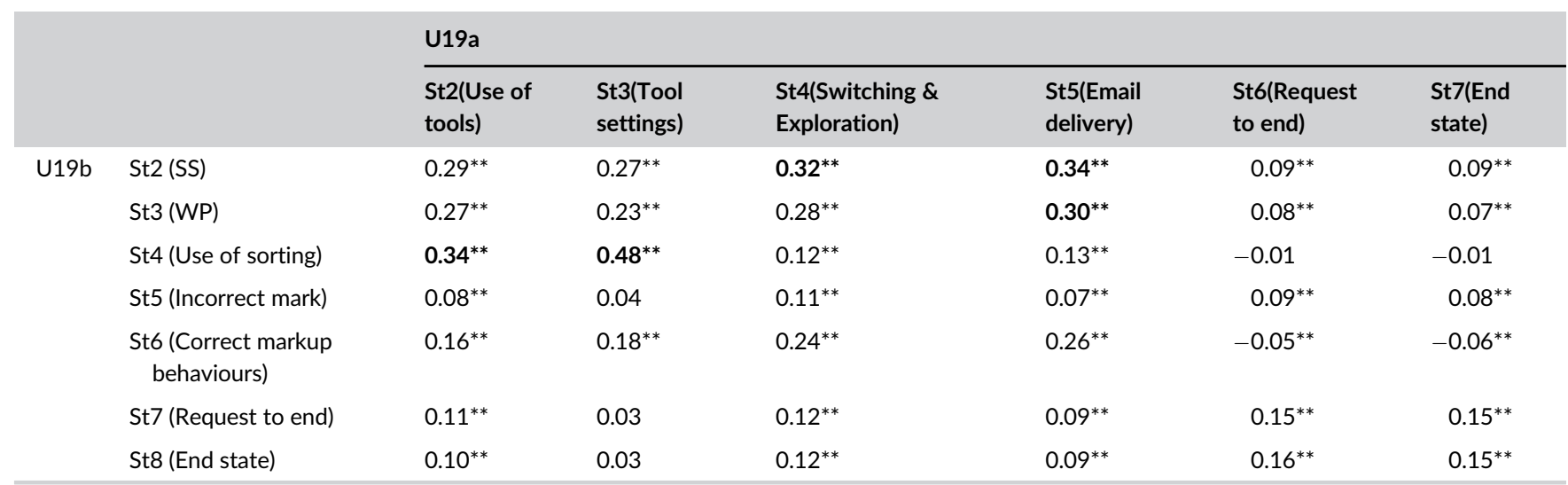

Note: Values greater than or equal to 0.30 are in bold.

${ }^{* *} p<0.01$.

\subsection{Associations between hidden states and performance}

To address the third research question, we explored the relationships between respondents' performance and extracted hidden states, as well as the transition between states by each item. For U19a, the correct and incorrect groups had 882 and 458 respondents, respectively. For U19b, the groups with scores of 0, 1, 2, 3, and 4 had 437, 31, 113, 189 , and 585 respondents, respectively. The average frequencies of states for two groups in U19a are shown in Figure 5 and the frequencies for five groups in U19b are in Figure 6, in which asterisks indicate the significance test results. The figures for state transitions of two items are attached in Appendix D.

As Figure 5 shows, in U19a, the correct group had a higher frequency than the incorrect group in four states (State 2, State 3, State 4 , and State 5). According to these states' interpretations, the correct group was found to use tools more frequently and switched between environments more often, which may indicate their repeatedly checking behaviours in the solving process. The correct group was also found more likely to execute key actions (i.e., actions that are highly related to credits). The incorrect group had a higher frequency in State 6 and State 7, which suggested the respondents were more likely to click cancel button when approaching to end the task. We further examined the frequency of transitions between states by each group. The transition from State 4 to State 6 was observed more often in the incorrect group, showing that these respondents more often chose to end the item immediately after some explorations. These explorations were likely to be non-targeted ones probably because of respondents' misunderstanding on the content of the task or lack of motivation to solve the item attentively. The incorrect group was also found to have more transitions from State 1 to State 6, suggesting that many respondents clicked the "NEXT" button immediately after starting the item, which resulted in a direct skip without any efforts in the task.

As Figure 6 shows, in U19b, the five performance groups significantly differed in the frequency of five states. Like the findings in U19a, the non-zero score groups (i.e., partial or full credit group) switched more often between environments than the zero-score group (i.e., fully incorrect group), reflected by more involvement in State 2 and State 3 . The frequency of State 4 varied significantly among groups with different scores. The full credit group (score $=4$ ) used the efficient tool (i.e., sorting) significantly more often than the partial credit groups (score $=1,2$, or 3 ). The fully incorrect group resulted in the lowest frequency in this state, on average lower than 1 , suggesting this group barely used the efficient tool when solving the task. Interestingly, the groups with partial and full credits had high frequencies in both State 6 (correct markup) and State 5 (incorrect markup). Their frequencies in State 5 were even higher than those in State 6, which might indicate the respondents retained marks for some incorrect rows in addition to the correct rows. Within our expectation, the group with no credit took fewer actions in State 5 and State 6 and their most actions occurred in State 5. Contrastively, in U19b, on average, the frequencies in State 7 (Request to end) and State 8 (End state) were resulted both as 1 and no significant differences were found between performance groups in these two states. It suggested that the hesitative behaviour clicking on the cancellation button were not obvious in this item.

When examining the difference in transition frequency between performance groups in U19b, we got the similar information as the state frequency. For the transition from State 1 (Start) to State 7 (Request to end), the frequency of the group with no credit was higher than that of other groups, suggesting a direct skipping behaviour in this item. The group with no credit also showed low transition frequencies between State 2 and State 3, indicating limited explorations in this item probably because of low motivation or lack of knowledge in understanding the task.

\subsection{Link between results of U19a and U19b}

The correlations of the state frequency are shown in Table 2. Because the starting states were adopted by all the respondents in each item, the correlation was always 1 . We excluded this state 


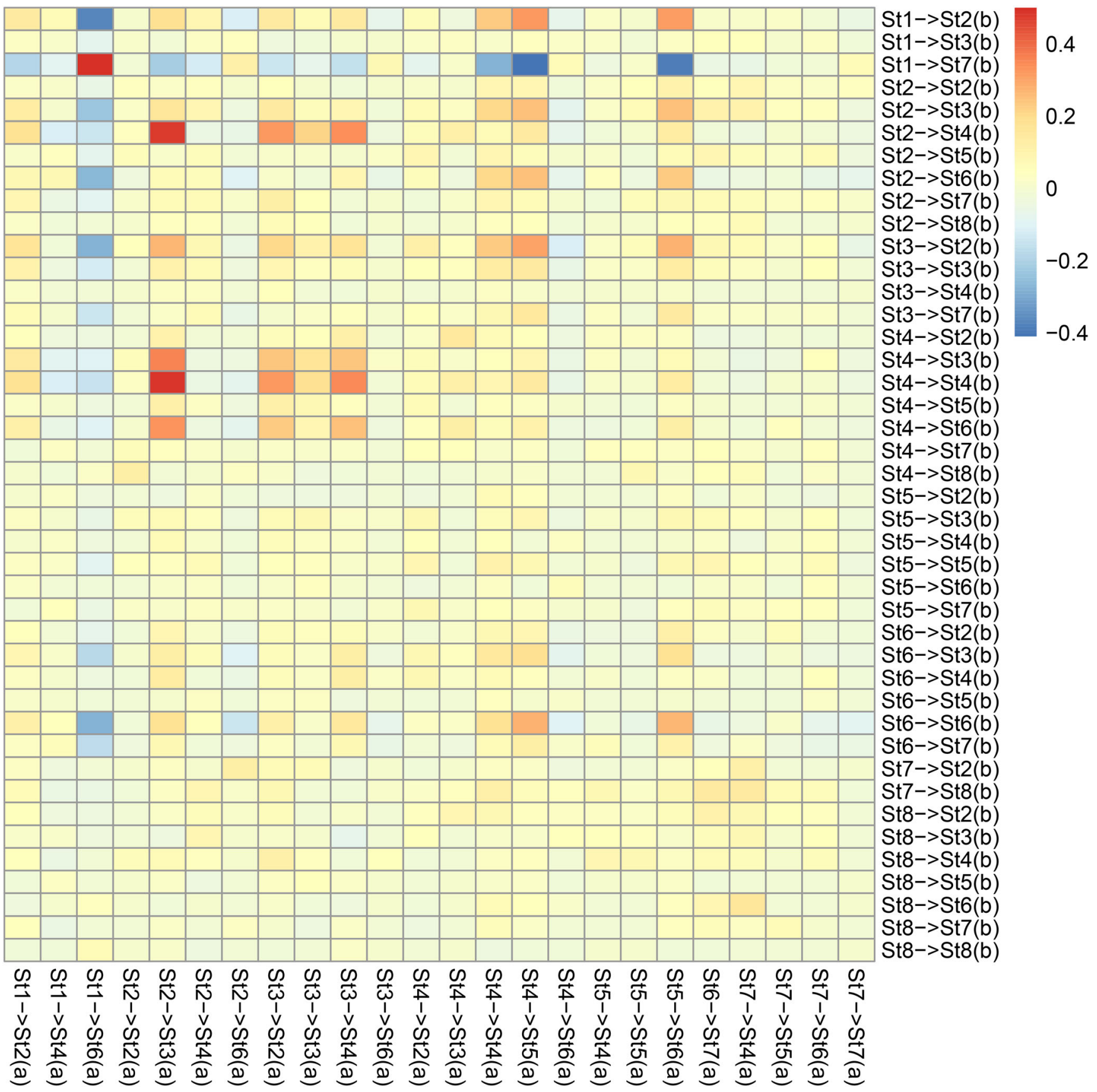

FIGURE 7 The heatmap of spearman correlations between state transition frequencies of two items. The horizontal axis corresponds to the transitions in U19a, shown by the suffix "(a)" in the horizontal axis labels. The vertical axis corresponds to transitions in U19b, denoted by the suffix "(b)" in the labels. The colour of each cell indicates the magnitude and direction of the correlation between the frequencies of two transitions from different items, as illustrated by the legend [Colour figure can be viewed at wileyonlinelibrary.com]

from both of the items when computing the correlation of state frequency. State 2 (Use of tools) and State 3 (Tool settings) in U19a are moderately correlated with State 4 in U19b (Use of sorting). These states revealed the use of efficient tools for solving the corresponding items. State 4 (Switching \& Exploration) and State 5 (Email delivery) in U19a has the correlations of about 0.3 with State 2 and State 3 in U19b, suggesting that the behaviour of switching between environments was moderately consistent in the two items.
The correlations of the state transition frequency are shown in the form of a heatmap (see Figure 7). The consistency of behaviours between the two items were illustrated more clearly. First, the transition from "Start" directly to "Request to end" had the highest correlation (0.50) between two items (St1 $\rightarrow$ St6 in U19a and St1 $\rightarrow$ St7 in U19b). And this transition in each item was negatively correlated to productive actions in another item, such as St $4 \rightarrow$ St 5 (email delivery after environment switch) and St5 $\rightarrow$ St6 (from email delivery to NEXT) in U19a, and St1 $\rightarrow$ St2 in U19b (switch to spreadsheet page 
after START). Second, strategic behaviours were positively correlated between items. For example, St2 $\rightarrow$ St3 in U19a (indicating the use of searching or sorting functions) was correlated with the transitions related to St4 (Use of sorting) in U19b. Also, St3 $\rightarrow$ St2 and St3 $\rightarrow$ St4 in U19a, the flagging action and environment switch after searching or sorting, were positively correlated with St2 $\rightarrow$ St4 (sorting after switching to spreadsheet page) and St4 $\rightarrow$ St4 (Use of sorting) in U19b.

\section{4 | DISCUSSION}

The response processes of problem-solving items contain rich information about respondents' behaviours and cognitive process in the digital tasks. In this study, we exploited the HMM on time-stamped action sequence data to identify the latent states and transitions between states underlying the problem-solving process in two PSTRE items in PIAAC. We found significant differences in behaviour patterns across latent states by response groups. The groups with correct responses in both items were found more engaged in tasks and more often to use efficient tools to solve problems, while the group with incorrect responses was found more likely to use shorter action sequences and exhibit hesitative behaviours. Consistent behavioural patterns were also identified through a correlation study across the two items.

This study demonstrated the advantages of HMM in process data analysis from both person-level and item-level perspectives. From the person-level aspect, HMM presented a deep insight of an underlying layer from the observed action sequences by segmenting each long problem-solving process into latent subtasks. This helped characterize response profiles and test-taking behaviours of people by different response groups (success and failure), thus providing potential features to cluster people with homogeneous behaviour patterns. For instance, Fincham et al. (2019) employed HMM to generate latent learning state sequences, based on which, learners were clustered into different learning strategy patterns. From the item-level aspect, the output from HMM blueprinted the transition path between latent states to help screening the abnormal behaviours that run out of item designers' expectation. For instance, respondents might not need to transit to the latent state of using tools (which is a compulsory step in predefined strategy created by item developers and content experts) but still could make correct responses following other strategies (e.g., scanning spreadsheet by rows with eyes). In such a case, the HMM could play an important role in examining the validity of a problem-solving item and help readjust the predefined strategies if they were too far away from the common individual problems-solving process.

Evidence-centered design (ECD) that was first introduced in Mislevy et al. (1999, 2003, 2006) presents the high-level models of the conceptual assessment framework (CAF) and four-process delivery architecture - examinee by examinee, the functions of selecting and administering tasks, interacting as required with the examinee to present materials and capture work products, and then evaluating responses from each task and accumulating evidence across theme. Evidence models under the CAF provide detailed instructions on how we should update our information about person-level variables given a performance in the form of examinees' work products from tasks. An evidence model contains two parts, which play distinct roles in the assessment argument: first, evidence rules describe how observable variables summarize an examinee's performance in a particular task from the work product that the examinee produced for that task; and second, the measurement model provides information about the connection between person-level variables and observable variables (Mislevy et al., 2003). In the current study, in fact, the work products are presented in the form of process data, specifically, time-stamped action sequences in our study. An application of HMM produced a hidden layer under the observational variables of work products. Looking ahead, a joint model containing both the person-level (response) variables and observable variables (i.e., action sequences and derived hidden states and transition probability from the HMM) is the machinery that effects probability-based accumulation and synthesis of evidence over task performances.

In this study, different behaviour characteristics were detected in successful and unsuccessful groups. These findings are consistent with the previous results of He and von Davier $(2015,2016)$, who applied ngrams model to explore the process data of the item U19a. In their study, respondents in the correct groups were found to be more likely to employ efficient tools, while those in the incorrect group were more likely to display cancellation-related behaviours and/or skip the items without executing any efforts. Unlike the n-gram model to segment the observable long action sequences into mini-sequences to facilitate the analysis, the application of HMM not only kept the action sequence as a complete set, but also provided latent states underneath the observable sequences, thus, made a further contribution to understanding the testtakers' behaviour in solving a digital task with various constrains and steps, especially in multiple environments related to spreadsheets and emails.

The latent states derived from the HMM corresponded to the subtasks disassembled from the problem-solving cognitive dimensions (e.g., setting sub-goals, using tools, acquiring information, and utilizing derived information) (OECD, 2012). For instance, the results related to tool use in different performance groups supported the theoretical importance of cognitive tools in assisting human problem solving (Jonassen \& Reeves, 1996). The sorting and/or searching functions in both items in this study could help narrow the searching range, thereby reducing cognitive load of problem solvers to save cognitive resources for higher-order thinking skills. The results of the current study are also aligned with the findings in Liu and Bera (2005) where problem-solvers in the higher performance levels were found more likely to make appropriate use of cognitive tools than those at the bottom level.

The application of HMM also disclosed a variety of transition path through latent states in the unsuccessful group, which could help pinpoint some reasons for respondents' failure. One possible reason could be the respondents' low motivation to solve the digital tasks. A typical low motivation of the skipping behaviour was following limited transition paths in latent states or simply from the state of start 
directly to the end. The low motivation might reduce an allocation of attentional resources to the task (Weith, 2005), thus resulted in a poor performance. An increasing number of recent studies have paid attentions to the association between behaviour patterns and motivations by different performance groups, mostly focused on using observable variables though. For instance, Sahin and Colvin (2020) suggested to combine information on the time spent on task with information on the number of performed actions to classify responses as rapid, disengaged responses. Ulitzsch, $\mathrm{He}$, and Pohl (2021) proposed using sequence mining techniques by combining action sequences and time intervals between actions to understand the incorrect behavioural patterns on interactive tasks. It would be interesting to leverage these observable variables with the latent states and transitions from the HMM application in the future studies.

\section{1 $\quad$ Limitations and future directions}

Some limitations also worth merit in this study. First, the extracted HMM states were interpreted according to actions with high emission probability. The validity of the latent states and transitions between states would be recommended for a further check with item developers and content experts. It was noted that the actions with low probabilities were not discussed in the current study, which might lead to ignorance of some useful information in special cases (e.g., some meaningful behaviour patterns that only occurred in a limited number of respondents).

Second, the two problem-solving tasks used in this study were relatively easy items with an over $60 \%$ correct rate (including partial credits) among respondents. The problem-solving strategies in these items were rather straightforward compared with the complex items. He et al. (2019) found that the task difficulty could lead to different degrees of complexity in forming problem-solving strategies, thus impacting respondents' behaviours. It would be interesting to expand the difficulty range in a future study to evaluate how respondents' behaviours would change under different situations. Further, more evidence is expected to be collected from multiple items across the PSTRE domain. A refined HMM approach would be valuable to generalize findings across multiple tasks in a further study.

Finally, because the current study only focused on using the process data from one participating country in PIAAC, the culture and country effects might not be revealed. An interesting direction for the future work could be leveraging covariates (e.g., age, gender, country, and low- and high- basic skills in literacy and numeracy) into the HMM to compare the different behavioural patterns in transitions and emission probabilities of latent states.

\section{5 | CONCLUSIONS}

This study presents the value of data-driven approach of HMM to reveal latent states and transitions underneath the observable action sequences to provide deeper insights of behavioural patterns in complex problem-solving tasks. Note that the HMM method is not restricted to time-stamped action sequences from complex problemsolving tasks but might be employed to understand behavioural patterns in various psychological assessment and digital learning settings with interactive modes such as collaborative, game- and simulation-based tasks, scenario-based environments, or learning apps. As such, the present study may serve as a blueprint for researchers seeking a better understanding of behavioural patterns in various types of interactive tasks. The application of HMM in process data analysis holds promise in better visualizing and disassembling the problem-solving process into latent subtasks, which could be helpful in evaluating the mastery of subskills from the complex problem-solving competence. As showcased in the present study, combining insights gained from the HMM application and expert-predefined sequences may support examining the item validity and complementing insights into different aspects of behavioural patterns and is therefore strongly advised.

\section{ACKNOWLEDGEMENT}

The authors thank the OECD-PIAAC (Programme for the International Assessment of Adult Competencies) team for granting access to the data source and instruments in this study and thank the Center for Psychometrics, Statistics, and Data Science and the Center for Global Assessments at the Educational Testing Service for their support in this research project. This project is partially supported by National Science Foundation grants IIS1633353 and by National Natural Science Foundation of China (32071091).

\section{CONFLICTS OF INTEREST}

The authors claim there has no known conflict of interest to disclose.

\section{ENDNOTES}

${ }^{1}$ In PIAAC, the item U19b was originally scored in a polytomous way as 0 - no credit (no correct rows marked), 1 - half credit (partially correct), and 2 - full credit (all correct) based on the number of correctly marked rows.

${ }^{2}$ The raw process data are openly available from the OECD website: http://www.oecd.org/skills/piaac/data/piaaclogfiles/.

${ }^{3}$ Each sorting option (i.e., change the sorting column name of sequence of order) was recoded as one single action.

\section{PEER REVIEW}

The peer review history for this article is available at https://publons. com/publon/10.1111/jcal.12559.

\section{DATA AVAILABILITY STATEMENT}

The raw process data that support the findings of this study are openly available from the OECD website: http://www.oecd.org/skills/ piaac/data/piaaclogfiles/, while the corresponding item documentation is not publicly available and is granted access upon reasonable request and with the permission of the OECD. 


\section{ORCID}

Yue Xiao (D) https://orcid.org/0000-0002-2836-2906

Qiwei He (D) https://orcid.org/0000-0001-8942-2047

Bernard Veldkamp (DD https://orcid.org/0000-0003-3543-2164

Hongyun Liu (D) https://orcid.org/0000-0002-3472-9102

\section{REFERENCES}

Akaike, H. (1973). Information theory and an extension of the maximum likelihood principle. In B. N. Petrov \& F. Csaki (Eds.), Second international symposium on information theory (pp. 267-281). Akadémiai Kiadó.

Arieli-Attali, M., Ou, L., \& Simmering, V. R. (2019). Understanding test Takers' choices in a self-adapted test: A hidden Markov modeling of process data. Frontiers in Psychology, 10, 83. https://doi.org/10.3389/ fpsyg.2019.00083

Bennett, R. E., Persky, H., Weiss, A. R., \& Jenkins, F. (2007). Problem solving in technology-rich environments. A report from the NAEP technologybased assessment project, research and development series (NCES 2007-466). National Center for Education Statistics.

Bennett, R. E., Persky, H., Weiss, A., \& Jenkins, F. (2010). Measuring problem solving with technology: A demonstration study for NAEP. The Journal of Technology, Learning and Assessment, 8(8), 1-44. https:// ejournals.bc.edu/index.php/jtla/article/view/1627

Bergner, Y., Walker, E., \& Ogan, A. (2017). Dynamic Bayesian network models for peer tutoring interactions. In A. A. von Davier, M. Zhu, \& P. C. Kyllonen (Eds.), Innovative assessment of collaboration (pp. 249268). Springer.

Biswas, G., Jeong, H., Kinnebrew, J. S., Sulcer, B., \& Roscoe, R. (2010). Measuring self-regulated learning skills through social interactions in a teachable agent environment. Research and Practice in Technology Enhanced Learning, 5(02), 123-152. https://doi.org/10.1142/ S1793206810000839

Boyer, K. E., Phillips, R., Ingram, A., Ha, E. Y., Wallis, M., Vouk, M., \& Lester, J. (2011). Investigating the relationship between dialogue structure and tutoring effectiveness: A hidden Markov modeling approach. International Journal of Artificial Intelligence in Education, 21(1-2), 6581. https://doi.org/10.3233/JAI-2011-018

Corbett, A. T., \& Anderson, J. R. (1994). Knowledge tracing: Modeling the acquisition of procedural knowledge. User Modeling and User-Adapted Interaction., 4(4), 253-278.

Damashek, M. (1995). Gauging similarity with n-grams: Languageindependent categorization of text. Science, 267(5199), 843-848. https://doi.org/10.1126/science.267.5199.843

Dutilh, G., Wagenmakers, E., Visser, I., \& van der Maas, H. L. J. (2010). A phase transition model for the speed-accuracy trade-off in response time experiments. Cognitive Science, 35(2), 211-250. https://doi.org/ 10.1111/j.1551-6709.2010.01147.x

Fincham, E., Gaševi'c, D., Jovanovi'c, J., \& Pardo, A. (2019). From study tactics to learning strategies: An analytical method for extracting interpretable representations. IEEE Transactions on Learning Technologies, 12(1), 59-72. https://doi.org/10.1109/TLT.2018.2823317

Forney, G. D. (1973). The viterbi algorithm. Proceedings of the IEEE, 61(3), 268-278. https://doi.org/10.1109/PROC.1973.9030

Ghahramani, Z. (2001). An introduction to hidden Markov models and Bayesian networks. In Hidden Markov models: applications in computer vision (pp. 9-41). World Scientific Publishing.

Goldhammer, F., Naumann, J., \& Keßel, Y. (2013). Assessing individual differences in basic computer skills. European Journal of Psychological Assessment, 29(4), 263-275. https://doi.org/10.1027/1015-5759/ a000153

Goldhammer, F., Naumann, J., Rölke, H., Stelter, A., \& Tóth, K. (2017). Relating product data to process data from computer-based competency assessment. In D. Leutner, J. Fleischer, J. Grünkorn, \& E. Klieme (Eds.), Competence Assessment in Education (pp. 407-425). Springer.

Greiff, S., Wüstenberg, S., \& Avvisati, F. (2015). Computer-generated logfile analyses as a window into students' minds? A showcase study based on the PISA 2012 assessment of problem solving. Computers \& Education, 91, 92-105. https://doi.org/10.1016/j.compedu.2015. 10.018

Han, Z., He, Q., \& von Davier, M. (2019). Predictive feature generation and selection using process data from PISA simulation-based environment: An application of tree-based ensemble methods. Frontiers in Psychology, 10, 2461. https://doi.org/10.3389/fpsyg.2019.02461

He, Q., Borgonovi, F., \& Paccagnella, M. (2019). Using Process Data to Understand Adults' Problem-Solving Behaviour in the Programme for the International Assessment of Adult Competencies (PIAAC): Identifying Generalised Patterns Across Multiple Tasks with Sequence Mining. OECD Publishing; OECD Education Working Papers. http://doi. org/10.1787/650918f2-en

He, Q., Borgonovi, F., \& Paccagnella, M. (2021). Leveraging process data to assess adults' problem-solving skills: Identifying generalized behavioral patterns with sequence mining. Computers \& Education, 166, 104170. https://doi.org/10.1016/j.compedu.2021.104170

He, Q., \& von Davier, M. (2015). Identifying feature sequences from process data in problem-solving items with n-grams. In L. A. van der Ark, D. M. Bolt, W.-C. Wang, J. A. Douglas, \& S.-M. Chow (Eds.), Quantitative psychology research (pp. 173-190). Springer. https://doi.org/10. 1007/978-3-319-19977-1_13

He, Q., \& von Davier, M. (2016). Analyzing process data from problemsolving items with n-grams: Insights from a computer-based largescale assessment. In Y. Rosen, S. Ferrara, \& M. Mosharraf (Eds.), Handbook of research on technology tools for real-world skill development (pp. 750-777). IGI Global.

Hirschberg, D. S. (1975). A linear space algorithm for computing maximal common subsequences. Communications of the ACM, 18(6), 341-343. https://doi.org/10.1145/360825.360861

Hirschberg, D. S. (1977). Algorithms for the longest common subsequence problem. Journal of the ACM, 24(4), 664-675. https://doi.org/10. 1145/322033.322044

Jeong, H., Gupta, A., Roscoe, R., Wagster, J., Biswas, G., \& Schwartz, D. (2008). Using hidden Markov models to characterize student behaviors in learning-by-teaching environments. In B. P. Woolf, E. Aïmeur, R. Nkambou, \& S. Lajoie (Eds.), Intelligent tutoring systems. Lecture notes in computer science (Vol. 5091, pp. 614-625). Springer. https://doi.org/ 10.1007/978-3-540-69132-7_64

Jonassen, D. H., \& Reeves, T. C. (1996). Learning with technology: Using computers as cognitive tools. In D. H. Jonassen (Ed.), Handbook of research for educational communications and technology (pp. 693-719). Macmillan.

Konishi, S., Ando, T., \& Imoto, S. (2004). Bayesian information criteria and smoothing parameter selection in radial basis function networks. Biometrika, 91(1), 27-43. https://doi.org/10.1093/biomet/91.1.27

Kroehne, U., \& Goldhammer, F. (2018). How to conceptualize, represent, and analyze log data from technology-based assessments? A generic framework and an application to questionnaire items. Behaviormetrika, 45(2), 527-563. https://doi.org/10.1007/s41237-018-0063-y

Kruskal, W. H., \& Wallis, W. A. (1952). Use of ranks in one-criterion variance analysis. Journal of the American Statistical Association, 47(260), 583-621. https://doi.org/10.1080/01621459.1952.10483441

LaMar, M. M. (2018). Markov decision process measurement model. Psychometrika, 83(1), 67-88. https://doi.org/10.1007/s11336-0179570-0

Levy, R. (2014). Dynamic Bayesian network modeling of game-based diagnostic assessments (CRESST report 837). University of California, National Center for Research on Evaluation, Standards, and Student Testing (CRESST). 
Levy, R. (2019). Dynamic Bayesian network modeling of game-based diagnostic assessments. Multivariate Behavioral Research, 54(6), 771-794. http://doi.org/10.1080/00273171.2019.1590794

Li, F., Cohen, A., Bottge, B., \& Templin, J. (2016). A latent transition analysis model for assessing change in cognitive skills. Educational and Psychological Measurement, 76(2), 181-204. https://doi.org/10.1177/ 0013164415588946

Liao, D., He, Q., \& Jiao, H. (2019). Mapping background variables with sequential patterns in problem-solving environments: An investigation of US Adults' employment status in PIAAC. Frontiers in Psychology, 10, 646. https://doi.org/10.3389/fpsyg.2019.00646

Liu, M., \& Bera, S. (2005). An analysis of cognitive tool use patterns in a hypermedia learning environment. Educational Technology Research and Development, 53(1), 5-21.

Mann, H. B., \& Whitney, D. R. (1947). On a test of whether one of two random variables is stochastically larger than the other. The Annals of Mathematical Statistics, 18(1), 50-60. https://www.jstor.org/stable/ 2236101

Manning, C. D., \& Schütze, H. (2002). Foundations of statistical natural language processing. MIT Press.

Mislevy, R. J., Almond, R. G., \& Lukas, J. F. (2003). A brief introduction to evidence-centered design. ETS Research Report Series, 2003(1), 1-29. https://doi.org/10.1002/j.2333-8504.2003.tb01908.x

Mislevy, R. J., Steinberg, L. S., \&Almond, R. G. (1999). On the roles of task model variables in assessment design (CSE Tech. Rep. No. 500). University of California, Center for the Study of Evaluation, Graduate School of Education \& Information Studies.

Mislevy, R. J., Steinberg, L. S., Almond, R. G., \& Lukas, J. F. (2006). Concepts, terminology, and basic models of evidence-centered design. In D. M. Williamson, R. J. Mislevy, \& I. I. Bejar (Eds.), Automated scoring of complex tasks in computer-based testing (pp. 15-47). Lawrence Erlbaum Associates.

Oakes, M., Gaizauskas, R., Fowkes, H., Jonsson, W. A. V., \& Beaulieu, M. (2001). A method based on chi-square test for document classification. In D. H. Kraft, W. B. Croft, D. J. Harper, \& J. Zobel (Eds.), Proceedings of the 24th Annual International ACM SIGIR Conference on Research and Development in Information Retrieval (pp. 440-441). ACM.

OECD. (2009). PIAAC problem solving in technology-rich environments: A conceptual framework. OECD Publishing. https://doi.org/10.1787/ 220262483674

OECD. (2011). PIAAC conceptual framework of the background questionnaire main survey. OECD Publishing. http://www.oecd.org/skills/piaac/ PIAAC(2011_11)MS_BQ_ConceptualFramework_1\%20Dec\% 202011.pdf

OECD. (2012). Literacy, numeracy and problem solving in technology-rich environments: Framework for the OECD survey of adult skills. OECD Publishing. https://doi.org/10.1787/9789264128859-en

OECD. (2017). PIAAC 2012 item documentation. OECD Publishing. https:// doi.org/10.4232/1.12569

Pelánek, R. (2017). Bayesian knowledge tracing, logistic models, and beyond: An overview of learner modeling techniques. User Modeling and User-Adapted Interaction, 27(3), 313-350.

Rabiner, L. R. (1989). A tutorial on hidden Markov models and selected applications in speech recognition. Proceedings of the IEEE, 77(2), 257286. https://doi.org/10.1109/5.18626

Sahin, F., \& Colvin, K. (2020). Enhancing response time thresholds with response behaviors for detecting disengaged examinees. Large-scale Assessment in Education, 8(5), 1-24. https://doi.org/10.1186/s40536-020-00082-1
Schleicher, A. (2008). PIAAC: A new strategy for assessing adult competencies. International Review of Education, 54(5-6), 627-650. https:// doi.org/10.1007/s11159-008-9105-0

Shu, Z., Bergner, Y., Zhu, M., Hao, J., \& von Davier, A. A. (2017). An item response theory analysis of problem-solving processes in scenario-based tasks. Psychological Test and Assessment Modeling, 59(1), 109-131.

Stadler, M., Fischer, F., \& Greiff, S. (2019). Taking a closer look: An exploratory analysis of successful and unsuccessful strategy use in complex problems. Frontiers in Psychology, 10, 777. https://doi.org/10.3389/ fpsyg.2019.00777

Sukkarieh, J. Z., von Davier, M., \& Yamamoto, K. (2012). From biology to education: Scoring and clustering multilingual text sequences and other sequential tasks. ETS Research Report Series, 2012(2), i-43.

Tang, X., Wang, Z., He, Q., Liu, J., \& Ying, Z. (2020). Latent feature extraction for process data via multidimensional scaling. Psychometrika, 85, 378-397. https://doi.org/10.1007/s11336-020-09708-3

Ulitzsch, E., He, Q., Ulitzsch, V., Nichterlein, A., Molter, H., Niedermeier, R., \& Pohl, S. (2021). Combining clickstream analyses and graph-modeled data clustering for identifying common response processes. Psychometrika, 86, 190-214. https://doi.org/10.1007/s11336-020-09743-0

Ulitzsch, E., He, Q., \& Pohl, S. (2021). Using sequence mining techniques for understanding incorrect behavioral patterns on interactive tasks. Journal of Educational and Behavioral Statistics, Advance online publication, https://doi.org/10.3102/10769986211010467

van de Sande. (2013). Properties of the Bayesian knowledge tracing model. Journal of Educational Data Mining, 5(2), 1-10.

Visser, I., \& Speekenbrink, M. (2010). depmixS4: an R package for hidden Markov models. Journal of Statistical Software, 36(7), 1-21.

Viterbi, A. J. (1967). Error bounds for convolutional codes and an asymptotically optimum decoding algorithm. IEEE Transactions on Information Theory, 13(2), 260-269. https://doi.org/10.1109/TIT.1967.1054010

Wang, S., Yang, Y., Culpepper, S. A., \& Douglas, J. A. (2018). Tracking skill acquisition with cognitive diagnosis models: A higher-order, hidden markov model with covariates. Journal of Educational and Behavioral Statistics, 43(1), 57-87. https://doi.org/10.3102/1076998617719727

Weith, M. (2005). The influence of motivation on cognitive mechanisms involved in problem solving (Doctoral dissertation). http://www.pqdtcn. com/thesisDetails/8DEB1024F95C875CB58827371B34678A

Wilcoxon, F. (1945). Individual comparisons by ranking methods. Biometrics Bulletin, 1(6), 80-83.

Zhu, M., Shu, Z., \& von Davier, A. A. (2016). Using networks to visualize and analyze process data for educational assessment. Journal of Educational Measurement, 53, 190-211. https://doi.org/10.1111/ jedm.12107

\section{SUPPORTING INFORMATION}

Additional supporting information may be found online in the Supporting Information section at the end of this article.

How to cite this article: Xiao, Y., He, Q., Veldkamp, B., \& Liu, H. (2021). Exploring latent states of problem-solving competence using hidden Markov model on process data. Journal of Computer Assisted Learning, 37(5), 1232-1247. https://doi.org/10.1111/jcal.12559 